\title{
An active-shield method for the reduction of surface contamination in CUORE
}

M. Pedretti*, F. Alessandria ${ }^{\dagger}$, R. Ardito**, , C. Arnaboldi** F.T. Avignone III ${ }^{\S}$, M. Balata , I. Bandac ${ }^{\S}$, M. Baruccil, J.W. Beeman ${ }^{\dagger}$, F. Bellinit , C. Brofferio**, C. Bucci ${ }^{\top}$, S. Capelli**, L. Carbone**, S. Cebrian ${ }^{\S \S}$, M. Clemenza* $^{* *}$, C. Cosmelli*,

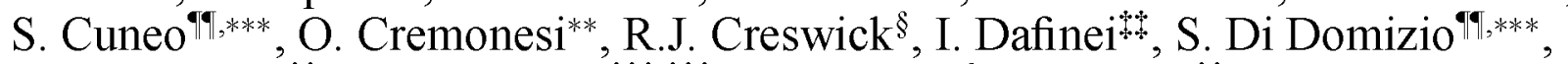

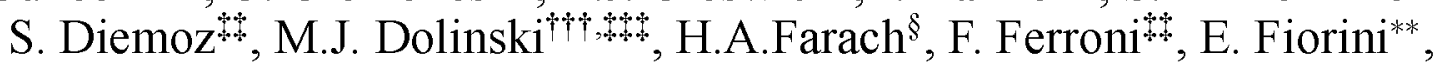

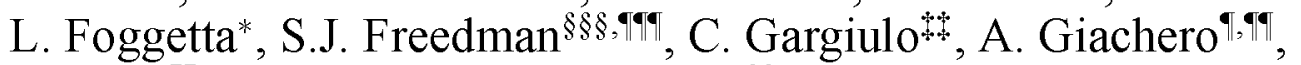

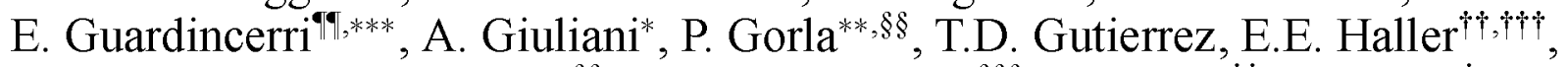
K.M. Heeger, I. G. Irastorza ${ }^{\S \S}$, Yu.G.Kolomenski ${ }^{\S \S}$, E. Longo*, G. Maier*, R. Maruyama, C. Martinez ${ }^{\S}$, S. Morganti ${ }^{+}$, S. Nisi ${ }^{\ddagger}$, C. Nones**, E.B. Norman ${ }^{* *}$,

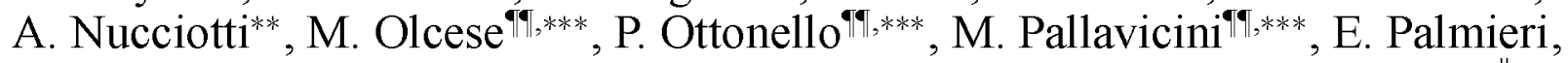
M. Pavan**, G. Pessina**, S. Pirro**, G. Pessina**, E. Previtali**, L. Risegarill, C. Rosenfeld ${ }^{\S}$, C. Salvioni*, S. Sangiorgio*, M. Sisti**, A.R. Smith ${ }^{\S \S}$, L. Torres ${ }^{* *}$, G. Venturall, M. Vignati ${ }^{*}$, N. Xu ${ }^{\S \S \S}$, C. Zarra and L. Zanotti**

*Dipartimento di Fisica e Matematica dell'Università dell'Insubriae

Sezione INFN di Milano, Como I-22100, Italy

${ }^{\dagger}$ INFN - Sezione di Milano, Milan I-20133, Italy

*** Dipartimento di Fisica dell'Università di Milano-Bicocca e Sezione di Milano dell'INFN, Milan I-2016, Italy

$\$$ Dipartimento di Ingegneria Strutturale del Politecnico di Milano, Milano I-20133, Italy

$\S$ Department of Physics and Astronomy, University of South Carolina, Columbia, South Carolina,29208, USA

'Laboratori Nazionali del Gran Sasso, I-67010, Assergi (L'Aquila), Italy

"Dipartimento di Fisica dell'Università di Firenze e

Sezione di Firenze dell'INFN, Firenze I-50125, Italy

${ }^{\dagger}$ Materials Science Division, Lawrence Berkeley National Laboratory, Berkeley, CA 94720, USA

*Dipartimento di Fisica dell'Università di Roma e Sezione di Roma 1 dell'INFN, Roma I-16146, Italy

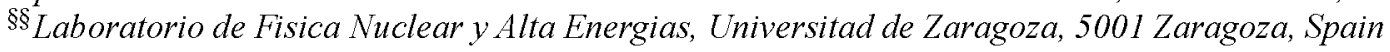

"I Dipartimento di Fisica dell'Università di Genova e Sezione di Genova dell'INFN, Genova I-16146, Italy

*** Sezione di Genova dell'INFN, Genova I-16146, Italy

$\dagger^{\dagger \dagger}$ Department of Materials Science and Mineral Engineering,

University of California, Berkeley, CA 94720 USA

$\$$ Lawrence Livermore National Laboratory, Livermore, California, 94550, USA

$\S \S$ Nuclear Science Division, Lawrence Berkeley National Laboratory, Berkeley, CA 94720, USA

"T Department of Physics, University of California, Berkeley, CA 94720, USA

University of Wisconsin, Madison, Wisconsin, USA

Laboratori Nazionali di Legnaro, Via Romea 4. I-35020 Legnaro (Padova),Italy

\begin{abstract}
The main goal of the CUORE experiment is to search for the neutrinoless double beta decay of ${ }^{130} \mathrm{Te}$. As it is a rare nuclear decay, the sensitivity of the experiment strongly depends on the background level in the transition energy region. In this paper we describe the $R \& D$ work performed to develop an active method for the reduction of radioactive background in CUORE. The idea is to reject events originated by surface contamination in large mass bolometric detectors by using bolometers sensitive to surface events. Results obtained with the first prototypes and tests made with large mass surface sensitive bolometers will be reported.
\end{abstract}

Keywords: cryogenic detector, bolometer, double beta decay, neutrino

PACS: $14,60 \mathrm{Pq}, 14.60 \mathrm{St}, 07.57 . \mathrm{Kp}, 95.55 . \mathrm{Rg}, 07.20 . \mathrm{Mc}$

CP897, Topical Workshop on Low Radioactivity Techniques, LRT 2006, edited by P. Loaiza

๑ 2007 American Institute of Physics 978-0-7354-0402-1/07/\$23.00 


\section{INTRODUCTION}

Experiments searching for rare events, like neutrinoless double beta decay $(0 v \mathrm{DBD})$ and dark matter, need to achieve very low background levels. In the $0 v \mathrm{DBD}$ case, for example, researchers have to find evidence of a peak at the energy transition of the decay over background fluctuations [2]. $0 \mathrm{vDBD}$ is a rare spontaneous nuclear transition where a nucleus $(A, Z)$ changes, with no 2 neutrinos emission, the nuclear charge of two units maintaining the same mass number and becoming an $(A, Z+2)$ nucleus:

$$
\text { 0vDBD : } \quad(A, Z) \rightarrow(A, Z+2)+2 e^{-}
$$

An experimental confirmation of this decay mode will constitute an important step for a better comprehension of neutrino physics and for the study of elementary particle physics beyond the SM. $0 v \mathrm{DBD}$, if exists, has a very long lifetime (larger than $10^{18}-10^{22}$ ): its peak signature can therefore be hidden by the presence of unavoidable traces of other radioisotopes with similar transition energies and lifetimes that are even 10 orders of magnitude shorter. This makes a dangerous background source.

\section{The CUORE experiment}

The CUORE (Cryogenic Underground Observatory for Rare Events) experiment [1] will start taking data from the beginning of 2011 at the Underground National Laboratory of Gran Sasso (LNGS). The CUORE detector will be an array of 988 bolometers working in a dilution refrigerator. A bolometer is a low temperature detector sensitive to single particle interactions whose basic idea is to measure the energy lost by particles as a variation of temperature. It is composed by two main elements: the energy absorber, where particles interact, and the sensor, which measures the temperature variation and converts it in an electrical signal. In order to achieve good energy resolutions, the CUORE detector will contain also the source of the searched decay (calorimetric approach): the energy absorbers in CUORE will then be $760 \mathrm{~g} \mathrm{TeO}_{2}$ crystals for a total mass of $741 \mathrm{~kg}$. As the isotopical abundance of ${ }^{130} \mathrm{Te}$ is about $34 \%$, the use of enriched crystals could improve the experimental sensitivity of CUORE, although it is not strictly needed.

The energy transition of the $0 \nu \mathrm{DBD}$ of ${ }^{130} \mathrm{Te}$ is positioned at $2530.3 \mathrm{keV}$, between the full peak and the Compton edge of the ${ }^{208} \mathrm{Tl}$ gamma line: this is, at least in principle, a low background energy region. A background of less than 0.01 counts $/ \mathrm{keV} / \mathrm{kg} / \mathrm{y}$ near the energy region of interest is required for CUORE in order to reach the desidered sensitivity, allowing to probe the inverse hierarchy region of effective Majorana neutrino mass.

\section{Interpretation of present Cuoricino background}

The feasibility of the CUORE experiment has been demonstrated by the Cuoricino experiment [3], whose detector can be seen as a single tower of CUORE. It is an array of $62 \mathrm{TeO}_{2}$ bolometers for a total sensitive mass of about 40 $\mathrm{kg}$ and it is by far the most massive running cryogenic detector for the search of rare events. It has been taking data since 2003 and the counting rate in the region of neutrinoless double beta decay is $0.18 \pm 0.01$ counts keV ${ }^{-1} \mathrm{~kg}^{-1}$ year $^{-1}$. The present best interpretation of this continuous background, coming from Montecarlo simulations of the whole detector, is that it is mainly due to degraded alphas coming from the passive materials that face the crystal absorbers, as the copper structures holding the detectors. This shows that Cuoricino is not only a powerful experiment that is giving important results on DBD, but it is also a good instrument to obtain information in view of the CUORE project. Moreover, to make an R\&D work for CUORE, this model of the Cuoricino background allows us to study the 3-4 MeV region of the energy spectra in order to understand if new tests feature a reduction of the continuous background due to degraded alphas. This is important because in the R\&D CUORE test facility the background at energies lower than the ${ }^{208} \mathrm{Tl}$ line (then in the $0 \mathrm{vDBD}$ energy region) is dominated by gammas due to the surrounding set up, which is not optimized for low radioactivity measurements.

\section{SURFACE SENSITIVE BOLOMETERS}

Using a very simple approximation known as the monolithic model, in a bolometric detector the signal amplitude of thermal pulses due to an instantaneous release of energy $E$ in the absorber is proportional to the ratio of $E$ to the heat 
capacity $C$ of the bolometer, whereas the pulse time constant is given by the ratio of $C$ to the thermal conductance $G$ between the detector and the heat sink. Consequently, in order to achieve high and fast signals, it is important to use bolometers with small heat capacity. This is the reason why these detectors operate at low temperature $(\sim 10 \mathrm{mK}$ in our case) and use dielectric and diamagnetic materials as $\mathrm{TeO}_{2}$ crystals. Bolometric detectors are not sensitive to the type of interacting particle or to the impact point of particle interaction, and, for this reason, surface contaminations are a dangerous source of background. The basic idea behind the Surface Sensitive Bolometer (SSB) is to surround the main absorber with active shields, which are also bolometers and are thermally connected to the main Cuoricino-like bolometer [5]. In this way the SSB is able to distinguish among three main types of events:

- events characterized by particles coming from outside the entire detector and stopped by the active shields. This is the case, for example, of degraded alphas coming from passive detector materials;

- those characterized by particles coming from the surface of the main absorber or of the shields, like for example a decay due to surface contamination of the $\mathrm{TeO}_{2}$ crystal;

- events that happen in the bulk of the main absorber, as the case of neutrinoless double beta decay.

We remark that any kind of particle interaction will give coincident thermal pulses on both shields and main bolometers, as they are thermally connected.

Thermal pulses are read out by semiconductor thermistors (Neutron Transmutation Doped sensor, NTD) thermally coupled to the absorbers by epoxy glue. The main absorber and each of the shielding bolometers have their own thermistor, and each absorber-thermistor pair can be regarded as an independent detector. Thermometers are biased resulting in a static heat flux, determining therefore temperature differences between the thermistor and the absorber. These differences are generally much lower than the absolute temperatures of the detector components, therefore negligible. The main absorbers are linked to a copper frame heat sink through a set of PTFE (Polytetrafluoroethylene) stand-offs, and the copper frame is thermally coupled to the coldest point of the housing dilution refrigerator. A picture of a Ge SSB prototype is shown in fig. 1.
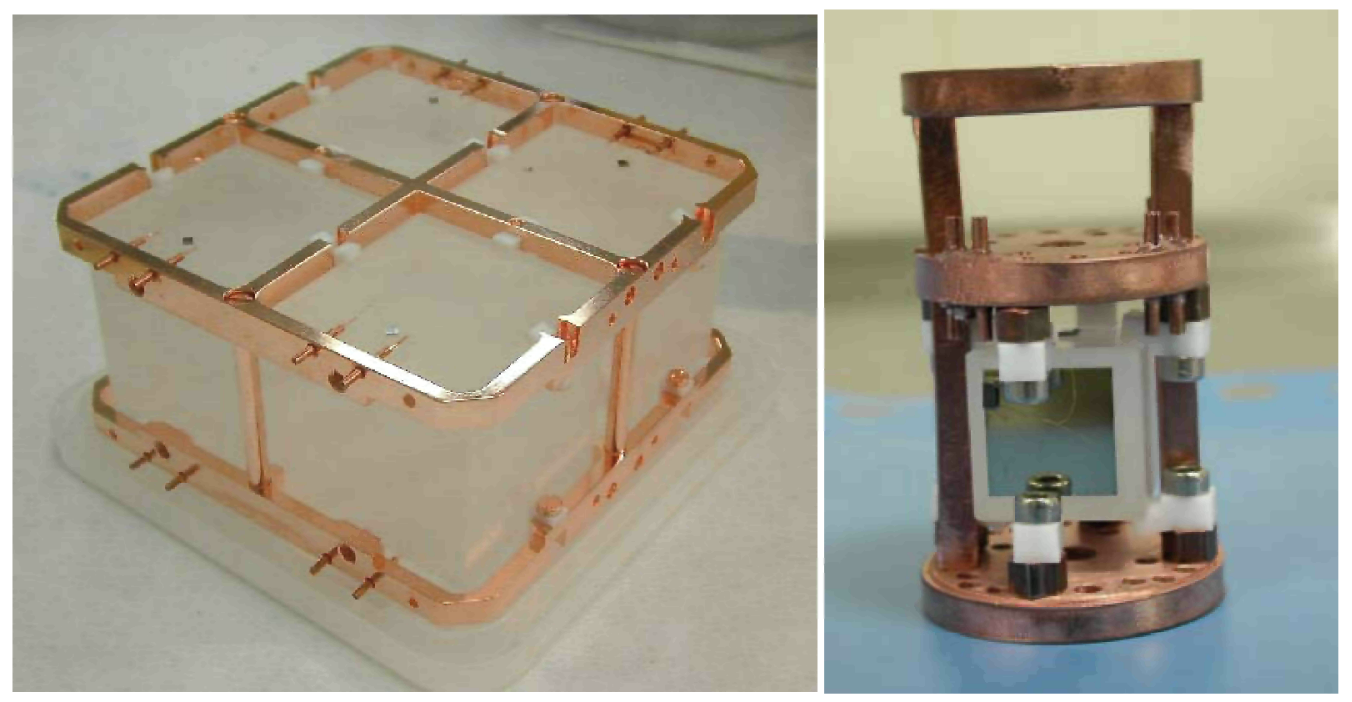

FIGURE 1. (Left: Picture of a single module of Cuoricino detector. It contains four $5 \times 5 \times 5 \mathrm{~cm}^{3} \mathrm{TeO}_{2}$ bolometric detectors. Right: Picture of the first SSB prototype. The thin germanium bolometer is thermally connected to the main $\mathrm{TeO}_{2}$ bolometer, in this way an energy deposition produce two coincident thermal pulses, one on each NTD sensor.

The classes of events previously described can be distinguished by performing a pulse-shape comparison of coincident pulses allowing to make a rejection of surface events. Particles from materials outside the SSB release energy in the shielding bolometer. Because of the small heat capacity of the shield, due to its small thickness, the signal read by its thermistor will have a higher amplitude and faster rise time than the signal read by the thermistor attached to the main absorber. If, on the other hand, an event happens inside the main absorber, all the thermistors of the detector will read pulses with comparable amplitudes and rise times. It is therefore possible to separate events with distinct origins through a comparison in amplitude and shape (e.g. rise and decay times) of pulses among the different thermistors. 


\section{First prototypes at Insubria University}

The first prototype SSBs were assembled and tested at Insubria University in Como, Italy. The main absorber and the active shields may be of the same or different materials depending on physical requirements, high purity and appropriate thermal properties. The prototypes had three different active shields, $\mathrm{Ge}, \mathrm{Si}$, and $\mathrm{TeO}_{2}$ crystals, and all used a $\mathrm{TeO}_{2}$ crystal as the main absorber, as they were thought in view of the CUORE experiment. Each main absorber was sandwiched between two active shields, and the main absorber was thermally and mechanically coupled to the heat bath by four PTFE stand-offs. These tests were performed in an above-ground laboratory: therefore, in order to reduce cosmic rays interactions, small SSBs have been designed. For this reason, smaller main absorbers $\left(20 \times 20 \times 5 \mathrm{~mm}^{3}\right.$ for the Ge and Si SSBs and $20 \times 20 \times 8 \mathrm{~mm}^{3}$ for the $\mathrm{TeO}_{2} \mathrm{SSB}$ ) were used rather than the $50 \times 50 \times 50 \mathrm{~mm}^{3}$ cubes used in Cuoricino.

The shields were $0.5 \mathrm{~mm}$ thick for $\mathrm{Ge}$ and $\mathrm{TeO}_{2}$ and $0.3 \mathrm{~mm}$ for $\mathrm{Si}$, and thermally coupled to the $\mathrm{TeO}_{2}$ main absorber by four small epoxy spots of $1 \mathrm{~mm}$ diameter and $50 \mu \mathrm{m}$ thickness. The coupling was designed to minimize the risk of the shields becoming detached from the difference in thermal contractions at cryogenic temperatures. For $\mathrm{TeO}_{2}$ shields, the [001] axis of the main and shield absorbers must be aligned to match the thermal contraction at low temperatures. Four small Ge stand-offs (about $1 \mathrm{~mm}^{2}$ large and $50 \mu \mathrm{m}$ thick) were inserted between the $\mathrm{TeO}_{2}$ shields and the main absorber, with thermal coupling established by the same epoxy. These stand-offs were introduced in order to establish a more reproducible procedure for the shield-main absorber mechanical and thermal connections.

Each absorber, the main absorber and both shields, was thermally coupled to its own NTD Ge thermistor. The thermal coupling between absorbers and thermistors was established by six epoxy beads $(0.5 \mathrm{~mm}$ diameter and $50 \mu \mathrm{m}$ thickness) for the main absorbers and $\mathrm{Ge}$ and $\mathrm{Si}$ shields and one larger spot for $\mathrm{TeO}_{2}$ shields.

An external source of $\alpha$-particles was used to test the SSB. The $\alpha$ source was a piece of copper strip implanted with ${ }^{224} \mathrm{Ra}$ nuclides. ${ }^{224} \mathrm{Ra}$ emits $\alpha$-particles with a half life of 3.66 days in equilibrium with its $\alpha$ and $\beta$ emitting daughters. The main $\alpha$ lines are at 5.68,6.29,6.78 and $8.78 \mathrm{MeV}$. Two other weak lines sum up to $6.06 \mathrm{MeV}$. A $\beta$ electron is emitted on average $0.3 \mu$ s before the $8.78 \mathrm{MeV} \alpha$ particle.

The typical operating temperature was $\sim 25 \mathrm{mK}$, corresponding to a thermistor resistance of $\sim 10 \mathrm{M} \Omega$. The main purpose of the experiment was to verify and understand the surface event discrimination capabilities of the detector and energy resolution was not optimized.

In fig. 2 an amplitude scatter plot obtained with the Ge SSB is shown. It is obtained by plotting the pulse amplitude measured by the sensor on one shielding bolometer versus the amplitude of the corresponding pulse obtained on the main bolometer. In this plot two different bands are evident. The steeper one corresponds to events that release energy in the shielding detectors whereas the lower one to particle interactions in the $\mathrm{TeO}_{2}$ bulk. For these reason the first region in the scatter plot is named "surface event band" and the second one "bulk event band". Bulk events are mainly due to natural $\gamma$ radioactivity and cosmic muons passing the $\mathrm{TeO}_{2}$ absorber. The points in the area between the two previously described regions are "mixed" events generated by interactions that release energy on both the active shield and the main absorber like for example cosmic muons interactions.

Another possibility in order to distinguish the origin of events is to use the pulse shape of coincident pulses. In fact, as already explained, surface events could be recognized by faster pulses on the active shieldings. Fig. 2 (right) shows the distribution of the rise time of pulses read out by the sensor on the shield that, as specified by the inset of the same figure, allows the discrimination of the two regions in the scatter plot.

These tests demonstrated the power of this active method for surface contamination rejection, but at the same time revealed the technical difficulties introduced by these new detectors. A totally active and totally shielding bolometer means a large increase in the number of electrical channels for the read out of all sensors: this is a serious issue in a cryogenic set-up, not only because of the heat power dissipated on the detectors, but also because these connections are not always safe and the loss of one of them could lead to an uncomplete understanding of the energy spectra of the main bolometer. In order to get rid of this problem, we studied with positive results in a Si SSB prototype the possibility to read out in parallel configuration the signals coming from the secondary bolometers. In this case the wires coming from the NTDs on the two silicon shields were connected in parallel. The obtained scatter plot is shown in fig. 3 (left).

The other major problem connected to the SSB idea is represented by the different thermal contractions between the shielding secondary bolometers and the main one. In the case of $\mathrm{Ge}$ and $\mathrm{Si}$ shields, this problem caused sometimes their mechanical disconnection. For this reason, even though Si slabs should be preferred from the economical and mechanical point of view, $\mathrm{TeO}_{2}$ represents the final choice for shielding, because using the same material of the main bolometer could solve the problem of differential thermal contractions. 

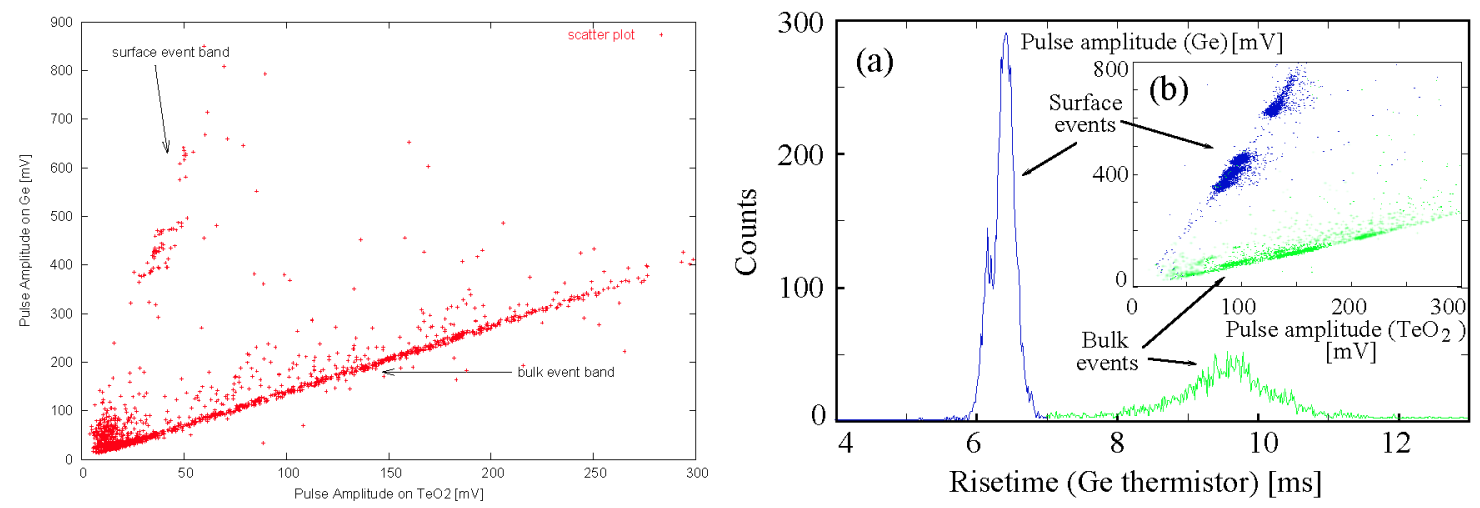

FIGURE 2. Left: Experimental scatter plot obtained from a SSB with Ge active shields. Right: rise time distribution for pulses read out by the NTD sensor on a Si secondary bolometer. Inset: scatter plot obtained with a Si SSB. The different colors evidence the contribution due to fast and slow signals.
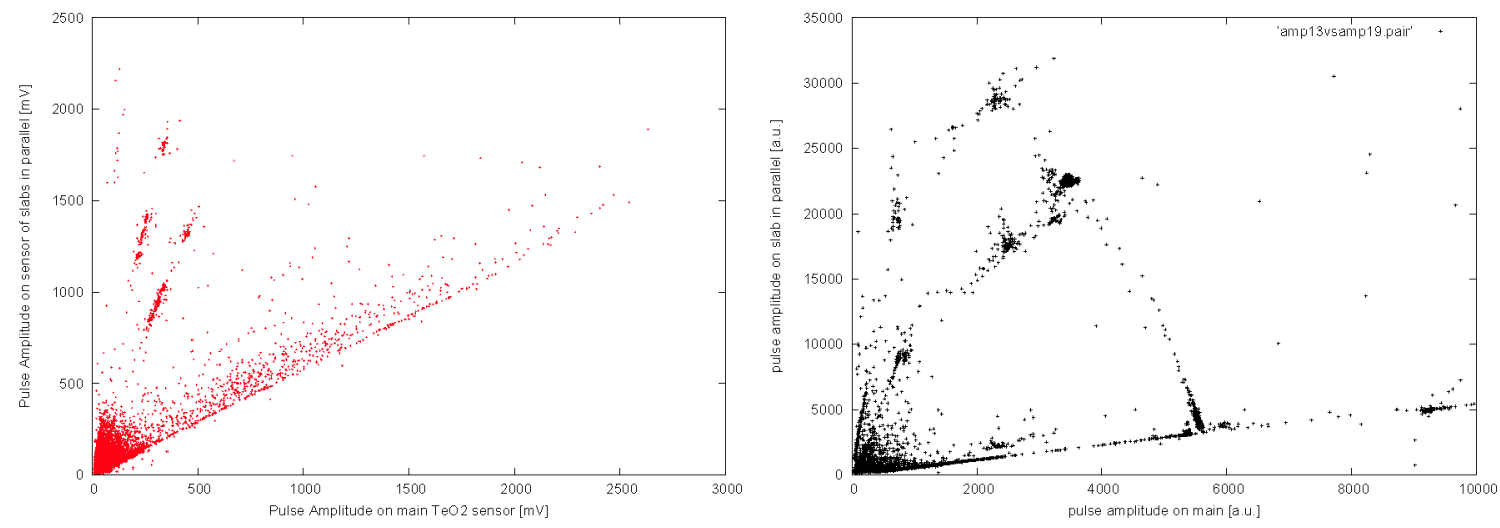

FIGURE 3. Left: scatter plot of the silicon SSB with the sensors on the two active shields connected in parallel. Right: scatter plot of a large mass Si SSB tested at LNGS. The complexity of this plot is due to the parallel read out and to the large amount of contaminations present on the silicon shields.

\section{Large scale test at LNGS and future test}

A large scale test was recently realized in the Hall C of LNGS, where a test facility for the CUORE R\&D is present. The main goal of this test was to prove the technical feasibility on real size detectors and not a result on background reduction. The used array of three planes was built according to the new design of CUORE (which implies a reduction in the amount of passive materials facing the detectors): four large SSB detectors were positioned in the bottom plane. Due to a delay in the delivery of $\mathrm{TeO}_{2}$ shields, the four SSB detectors were realized gluing on each face of the $5 \times 5 \times 5 \mathrm{~cm}^{3}$ crystals a Si shield $\left(50 \times 50 \times 0.3 \mathrm{~mm}^{3}\right)$. For three crystals the coverage was not exactly complete (an almost triangular area near each vertex was touched by the PTFE tips used to hold the crystal in the copper frame and then was not covered by active shields). Each shield was provided with its own NTD Ge thermistor, used to read out the thermal signal. The six thermistors on the shields were connected in parallel, as already tested in Como. In this way, for each $5 \times 5 \times 5 \mathrm{~cm}^{3}$ detector two channels were read out: one for the main absorber and one for the shields. Unfortunately, the main $\mathrm{TeO}_{2}$ crystals used for this test had different dimensions and the two smaller ones showed rather poor performances, due to the excess noise induced by their vibrations; for the other two, instead, a good energy resolution, compatible with that of Cuoricino detectors, was measured. Unluckily, only one of these good resolution detectors had readable shields because during the cooling the electrical connections to the shield thermistors of the other were lost. A long background measurement was used to understand the behavior of these detectors and to study the rejection efficiency for surface alpha contamination. The detector with complete read-out was used for an extensive study of the main features of the composite bolometer. In fig. 3 (right) the obtained scatter plot is presented. Here it 
is possible to identify different classes of events: those belonging to the main band; then the region of surface events, characterized by high signals on the shields channel; the region of so called mixed events due in this case to events that share energy both in the main and in the shielding absorbers; and events probably due to contaminations on the surface in between the $\mathrm{Si}$ and $\mathrm{TeO}_{2}$ absorbers. It is evident that the materials used in this test were largely contaminated, as no attention was paid to the to working in a clean condition during the assembly procedure.

A new powerful tool for the rejection of surface events emerged evidently in this test. In fact, in the read-out of just the NTD sensor on the main bolometer, pulses belonging to both the mixed and the surface regions in the scatter plot are characterized also by different decay times comparing to pulses belonging to the main events band. This could be evinced by comparing the decay time distribution for pulses read by the main absorber thermistor obtained with a usual Cuoricino detector (fig. 4 (left)) with the one (fig. 4 right) obtained with the SSB operated in this test. This means that shields act as shape modifiers for pulses read by the NTD thermometer on the main detector depending on the origin of events: such difference gives the possibility to use only the thermistor on the main bolometer and then allows a huge simplification in view of CUORE.
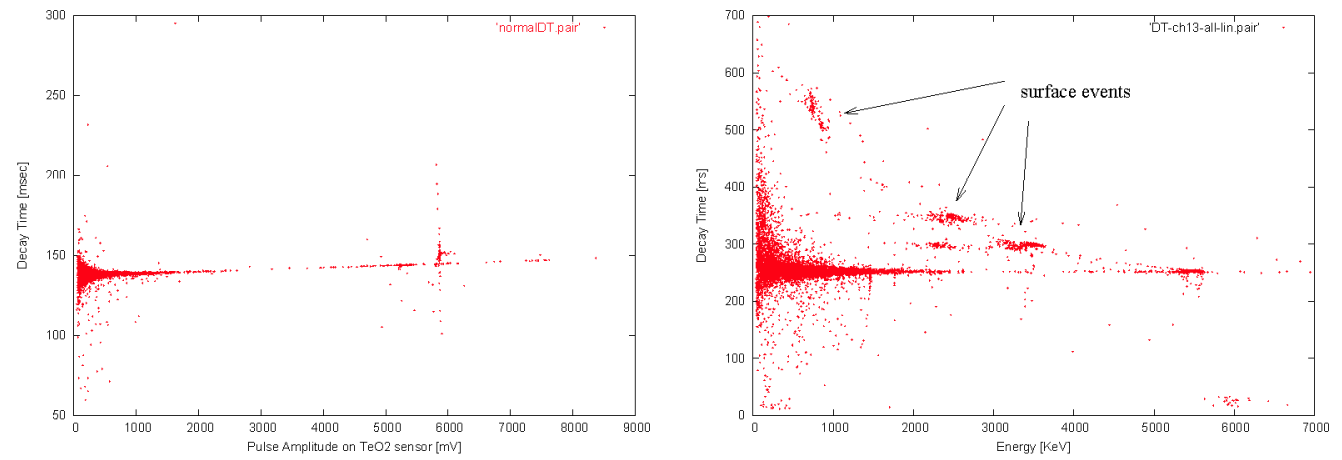

FIGURE 4. Left: decay time distribution of a usual Cuoricino detector. The pulses read by the main absorber thermistor are characterized by a decay time that is approximately constant at different energy. Right: decay time distribution for a SSB detector; a structure in the decay time is visible.

The power of this method is also confirmed by background results obtained by using the previously described discrimination analysis: the rejection of pulses through decay time parameters leads to a background level in the 3-4 $\mathrm{MeV}$ energy region compatible with the Cuoricino one, although with large errors due to the little statistics collected.

A new test with $\mathrm{TeO}_{2} \mathrm{SSBs}$ realized with attention to the cleanness of both the materials and the assembly procedure is just finished. The aim of this new test is to obtain a lower level of background and its comprehension. Its complete data analysis will be ready soon.

\section{ACKNOWLEDGMENTS}

We are pleased to acknowledge that this work has been partially supported by the ILIAS integrating activity (Contract No. RII3-CT-2004-506222) as part of the European Union FP6 programme in Astroparticle Physics, and by the PRIN2004 project "Large Mass, Low Noise Cryogenic Detectors to Search for Weak and Gravitational Processes" (Italian Ministery of Research and University).

\section{REFERENCES}

1. C. Amaboldi et al., Phys. Rev. Lett. 95, (2005) 142501

2. S. Elliott, J. Engel, J. Phys. G: Nucl. Part. Phys. 30 (2004) R183.

3. C. Amaboldi et al., Phys. Rev. Lett. 95, (2005) 142501.

4. M. Pedretti, Nucl. Phys. Proc. Suppl. 125, (2003) 380

5. L. Foggetta, et al., Appl. Phys. Lett, 86 (2005) 134106 
Copyright of AIP Conference Proceedings is the property of American Institute of Physics and its content may not be copied or emailed to multiple sites or posted to a listserv without the copyright holder's express written permission. However, users may print, download, or email articles for individual use. 